\title{
High density effective theory on the lattice
}

\section{A. Dougall}

Bergische Universität Wuppertal, 42119 Wuppertal, Germany

E-mail: ajd1@theorie.physik.uni-wuppertal.de

Long-range interactions in finite density QCD necessitate a non-perturbative approach in order to reliably map out the key features and spectrum of the QCD phase diagram. However, the complex nature of the fermion determinant in this sector prohibits the use of established Monte Carlo techniques that utilize importance sampling. Whilst significant progress has been made in the low density, high temperature region, this remains a considerable challenge at mid to high density. At large chemical potential, QCD can be approximated using high density effective theory which is free from the sign problem at leading order. We investigate the implementation of this theory on the lattice in conjunction with existing re-weighting techniques.

The XXV International Symposium on Lattice Field Theory

July 30 - August 42007

Regensburg, Germany 


\section{Introduction}

A key motivation for studying QCD at different temperatures and pressures arises from the need to understand the behaviour of matter in various extreme scenarios: the environment of intense heat and pressure that existed after the big bang, the ongoing experiments in heavy ion collisions, such as RHIC and ALICE, that aim to recreate a similar environment within the laboratory, and the conditions that exist at the centre of a neutron star where matter is cold and very densely packed. Furthermore, by investigating the different phases of QCD, one may gain further insight into confinement, chiral symmetry breaking and the nature of the QCD vacuum, which would contribute to our understanding of the structure of hadrons.

The behaviour of strongly interacting matter in thermal and chemical equilibrium is characterised by the temperature $T$ and quark chemical potential $\mu$. The main thermodynamic properties of QCD are summarised in the QCD phase diagram, shown in fig. 1.

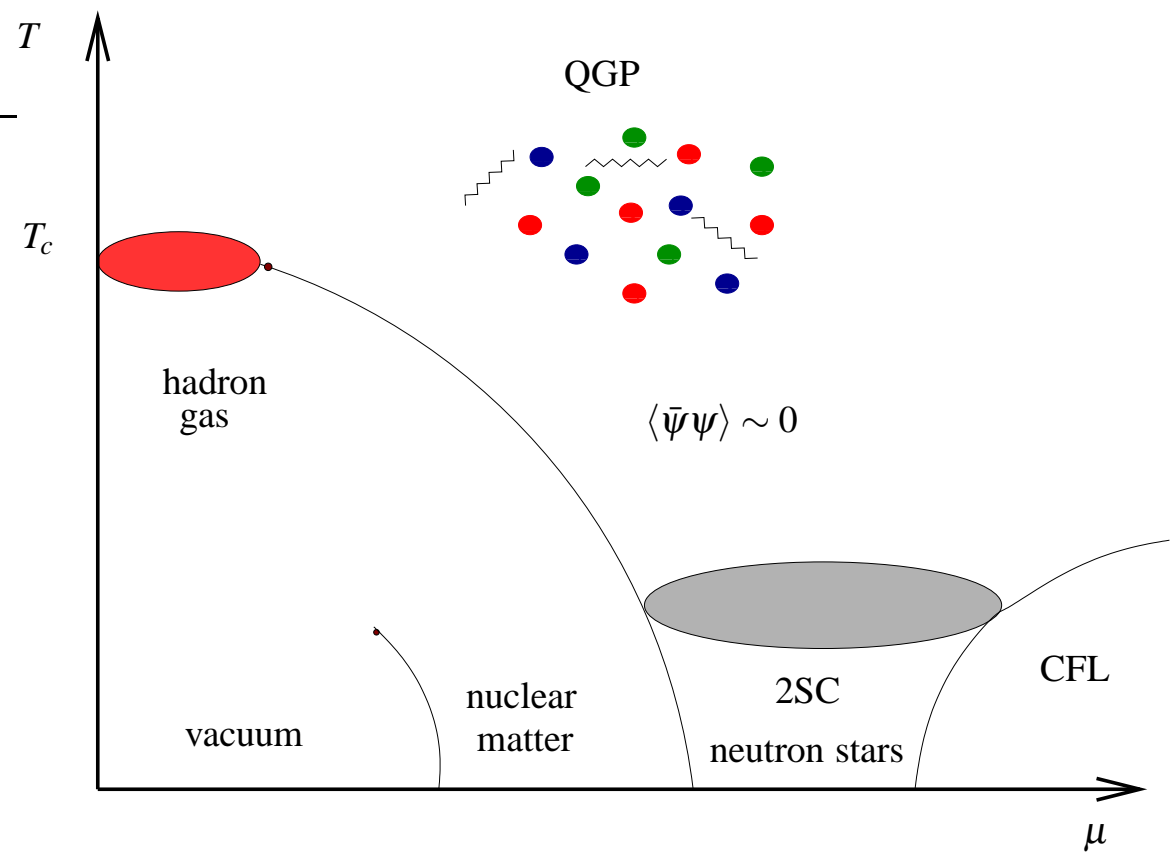

Figure 1: The QCD phase diagram.

Early attempts to map out the phase diagram revealed the existence of a deconfined phase and since then further details about the nature and location of the transition that separates these two phases have been uncovered. Some key features of the diagram include:

- confined hadronic matter and the QCD phase transition: We live in the phase where chiral symmetry is broken and there is colour confinement. At low temperatures, there is a boundary separating nuclear matter and the vacuum. As the temperature increases, a hadronic gas forms. If the temperature or pressure increases beyond certain limits, the system undergoes a phase transition, the nature of which depends on whether this is driven by an increase in temperature or density, or, an increase in both. 
- Quark-Gluon Plasma: In this phase QCD is deconfined and quarks and gluons are the fundamental degrees of freedom. In addition, chiral symmetry is approximately restored.

- temperature-driven phase transition: Located alongside the temperature axis of the phase diagram, the transition here was recently found to be a crossover [1], which places contraints on evolutionary models of the early universe.

- critical temperature: Located at the point where the phase transition crosses the temperature axis, the value of the critical temperature was recently calculated [2] to be $T=150(3)$ (3) MeV although since the phase transition is non-singular, there is no unique value for this quantity.

- density-driven phase transition: This transition, located along the chemical potential axis, is expected to be first order, however, it is currently difficult to reach due to its location in a region of intermediate density.

- critical points: A critical point is expected to occur along the first order phase transition as it moves towards a temperature-driven crossover. Lattice predictions [3] locate this point at $(T, \mu)=(162(2), 360(40)) \mathrm{MeV}$ (for $N_{t}=4$ and with large cut-off corrections expected). Recent work [4] indicates the existence of a triple-point connecting three different phases on the diagram, occuring at $\left(T, \mu_{q}\right)_{\text {tri }} \approx(137,300) \mathrm{MeV}$ (with large cut-off corrections).

In addition to the QGP phase, analytic calculations in the cold dense region of QCD have found alternative interaction channels leading to the prediction of colour superconductivity (2CS), where an attraction between pairs of quarks at the Fermi surface with opposite momenta leads to the formation of a condensate of quark cooper pairs. More recently, the phenomena of colour-flavour locking (CFL) was predicted [5], where diquark condensates form from three light quark flavours, breaking colour and flavour symmetries. Given that these interactions are highly non-perturbative, one would like to investigate these regions, together with the density-driven phase transition using lattice QCD. However, the sign problem has proven to be a considerable barrier to the investigation of densely packed matter.

\section{The sign problem}

In the lattice formulation, the expectation value of the operator $O$ is expressed in terms of the discrete Path Integral (PI). Having integrated over the fermion fields, the expectation value is given by

$$
\langle O\rangle=\frac{\int D U O(\operatorname{det} M)^{n_{f}} e^{-S_{G}}}{\int D U(\operatorname{det} M)^{n_{f}} e^{-S_{G}}}
$$

where $M$ is the fermion matrix and $n_{f}$ is the number of fermion flavours. This integral is computed numerically using standard Monte Carlo integration and in order to ensure that the background field configurations are sampled as efficiently as possible, importance sampling techniques are introduced, where the probability of selecting a particular configuration is weighted according to the distribution $P[U]=\frac{1}{Z}(\operatorname{det} M)^{n_{f}} e^{-S_{G}}$. The PI thus reduces to a sum over the operator evaluated on each background configuration, but we now have the constraint that $\operatorname{det} M$ be interpreted as a 
probability and therefore the Dirac operator must be positive. We can establish whether this is indeed the case by testing the " $\gamma_{5}$-hermiticity" property: $M^{\dagger}=\gamma_{5} M \gamma_{5}$. If this equation is satisfied, either $\operatorname{det} M$ is real or the eigenvalues are complex but paired, and the fermion determinant can be used to importance sample the background gauge configurations. In the case of QCD at non-zero density, the $\gamma_{5}$-hermiticity is spoiled by the sign of the chemical potential term and importance sampling is prohibited. Whilst progress has been made in the low density region, this remains a problem at high density and we are motivated to approach the problem using an effective theory.

\section{Effective theory approach to QCD at high density}

In this section we examine the physical characteristics of densely packed matter and introduce the high density effective theory (HDET) that incorporates the low-energy behaviour of the system. This theory was introduced and developed by Hong in a series of papers, the first of which is given in Ref. [6].

\subsection{The physical characteristics of densely packed matter}

When matter is added to a fixed volume the energy of the lowest available state increases due to the fermionic nature of quarks. Consequently, quarks at the Fermi surface of very densely packed matter have high momentum. An essential characteristic of such a system is that at low energy, high-momentum gluon exchange between quarks is suppressed because of the asymptotic nature of the QCD coupling. Typical Fermi surface interactions only change the quark momentum by a small amount and since these interactions are soft, $O\left(\Lambda_{\mathrm{QCD}}\right)$, they require a non-perturbative treatment. Furthermore, the low energy degrees of freedom are restricted since quarks at low momentum (within the Fermi sea) and antiquarks (within the Dirac sea) require high momentum interactions to excite them due to Pauli blocking (occupation) of the states above (see fig. 2). In essence, the only low energy dynamical degrees of freedom are the modes that lie near the Fermi surface which are quasi quarks and holes, together with soft gluons.

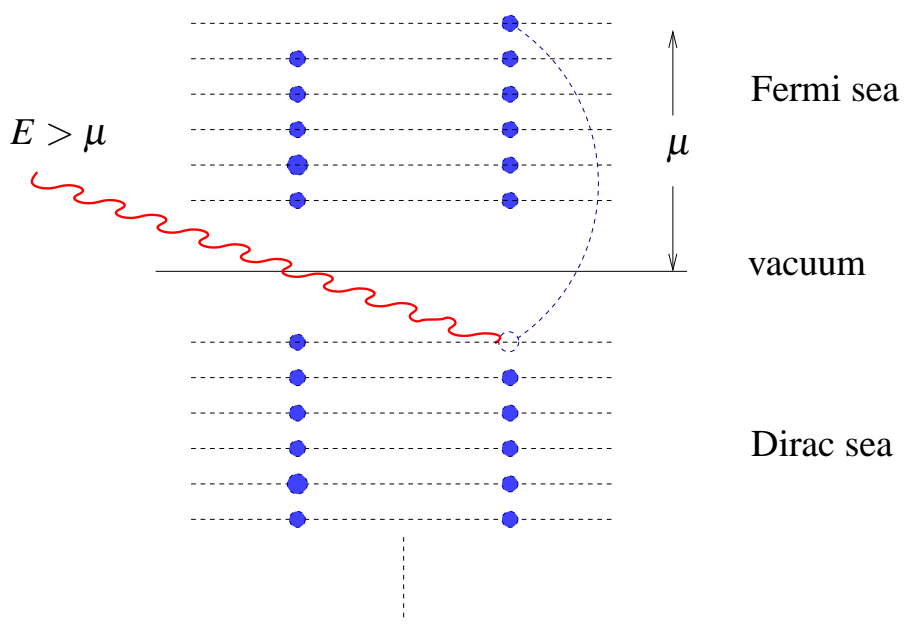

Figure 2: States within the Dirac sea and deep within the Fermi sea do not contribute to the low energy dynamics due to Pauli blocking. 


\subsection{Approximating dense QCD with an effective theory}

Analogously to HQET, since there is a high momentum scale which does not contribute to the low energy dynamics, in this case near the Fermi surface, the quark momentum can be decomposed into two pieces, $p^{\mu}=\mu v_{F}^{\mu}+l^{\mu}$, where $v_{F}$ is the Fermi velocity and the residual part $l^{\mu}$ is associated with the Fermi surface interactions. In order to access these modes, the full field is expanded into large and small components (quasi-quarks) to give

$$
\Psi(x)=\sum_{\overrightarrow{v_{F}}} e^{-i \mu v_{F} \cdot x}\left(\psi_{+}\left(\overrightarrow{v_{F}}, x\right)+\psi_{-}\left(\overrightarrow{v_{F}}, x\right)\right)
$$

where the large contribution to the momentum has been factored out leaving field components $\psi_{+}$and $\psi_{-}$that are velocity dependent and carry residual momentum $l$. The large and small components are defined respectively as

$$
\psi_{+}(x)=e^{i \mu v_{F} \cdot x} P_{+} \Psi(x) \text { and } \quad \psi_{-}(x)=e^{i \mu v_{F} \cdot x} P_{-} \Psi(x)
$$

with the projection operator $P_{ \pm}=\frac{1}{2}(1 \pm \vec{\alpha} \cdot \hat{v})$ and $\vec{\alpha}=\gamma^{0} \vec{\gamma}$. Since different modes of the quark field are characterised by an associated velocity, quarks with momentum modes that differ by more than the low energy exchanges decouple from each other and the Fermi surface (sphere) describing all the possible momentum modes can be split into patches, each labelled by an associated velocity. The size of a patch must be large enough to contain the low energy interactions which are $O\left(\Lambda_{Q C D}\right)$. It is possible to define the decomposition of the quark field into Fermi surface modes more precisely [7], thus avoiding the sum over patches, however, we do not use this approach since the resulting action cannot be discretised readily in its present form.

Using eqn. 3.1 the full QCD Lagrangian at high density can be re-expressed in terms of large and small components, with the $\psi_{+}$field describing quark modes at the Fermi surface and the $\psi_{-}$ field describing modes within the Dirac sea. Soft interactions cannot excite $\psi_{-}$so these modes are integrated out using the equations of motion to form a low energy effective theory that describes the strong interactions of modes at the Fermi surface. The tree-level effective Lagrangian of high density QCD [6] is given by

$$
L_{\text {hdet }}^{0}=\sum_{\mathbf{v}} \bar{\psi}_{\mathbf{v}}\left[i \gamma_{\|}^{\mu} D_{\mu}-\frac{\gamma_{0}\left(D_{\perp}\right)^{2}}{2 \mu} \sum_{n}\left(-\frac{i D_{\|}}{2 \mu}\right)^{n}\right] \psi_{\mathbf{v}}-\frac{1}{4} F_{\mu v}^{a} F^{a \mu v}
$$

where the parallel and perpendicular components of a generic four-vector $X^{\mu}$ are denoted $X_{\|}^{\mu}=$ $V^{\mu} X \cdot V$ and $X_{\perp}^{\mu}=X^{\mu}-X_{\|}^{\mu}$ respectively, with $V=\left(1, \overrightarrow{v_{F}}\right)$. The fields are now labelled by $\mathbf{v}$ instead of + , reflecting the fact that they are velocity dependent and the index $\mathbf{v}$ runs over the number of patches. The HDET Lagrangian is a systematic expansion in $1 / \mu$ and the coupling constant $\alpha_{s}$. There are coefficients in front of each term which contain information about the short distance physics which has been integrated out. These corrections are computed by matching the diagrams between the effective theory and the full theory at a given order in $\alpha_{s}$. The leading order term in the fermion action can be re-expressed as $i V \cdot D$ and is therefore spin-independent, whilst the NLO term can be written as a sum of spin-independent and spin-dependent terms.

The full dense QCD Lagrangian has now been re-expressed as an effective Lagrangian with $\mu$ appearing in the denominator. Provided that the residual momentum is much smaller than the 
chemical potential, this should serve as a good approximation to QCD at high density. Crucially, the sign problem does not occur at leading order in this theory [7], thus opening up the possibility for lattice calculations at high density.

\section{HDET on the lattice}

The strategy of the lattice calculation is to use the effective theory to look for evidence of $\mu$-dependence in a simple observable such as the plaquette. In formulating HDET on the lattice we highlight several important details concerning the inclusion of the NLO term, an approximation to the sum over velocities and the discretisation of the action.

\subsection{Reweighting the leading order calculation}

As discussed, the LO fermion operator is free from the sign problem and can therefore be used in the importance sampling of the field configurations. This is not possible in the case of the NLO term where the sign problem re-emerges. Since it is necessary to include this correction in order to have explicit $\mu$-dependence in the calculation, as well as enabling measurements at lower values of $\mu$, this term is incorporated into the measurement by reweighting the observable [8], such that

$$
\langle O\rangle=\frac{\left\langle O e^{-S_{\mathrm{NLO}}}\right\rangle}{\left\langle e^{-S_{\mathrm{NLO}}}\right\rangle} .
$$

\subsection{The sum over patches}

The effective Lagrangian contains a sum over patches, each of which correspond to a particular region of the Fermi surface with an associated unit velocity, $\vec{v}$. The physics within each patch is equivalent and therefore, to avoid a prohibitively expensive and complicated computation, we compute the Lagrangian for a single patch and then multiply by an overall factor to account for the sum. The number of patches covering the surface is given approximately by $N_{\text {patch }} \sim\left(4 \pi \mu^{2} / \Lambda_{\perp}^{2}\right)$ where $\Lambda_{\perp}$ is the cutoff on the transverse momenta. For example, given a chemical potential of $1.5 \mathrm{GeV}$, the number of patches is approximately 315 . The fermion part of the HDET Lagrangian (through NLO) becomes

$$
L_{\mathrm{hdet}}^{0}=N_{\mathrm{patch}} \bar{\psi}_{\mathbf{v}}\left[i \gamma_{\|}^{\mu} D_{\mu}-\gamma_{0} \frac{\left(D_{\perp}\right)^{2}}{2 \mu}\right] \psi_{\mathbf{v}}
$$

and we choose $\vec{v}=(0,0,1)$.

\subsection{Discretisation}

The discretisation of the HDET fermion action follows from the usual definition for the simplest form of the covariant derivative on the lattice. For $\vec{v}=(0,0,1)$, the LO term is given by

$$
N_{\text {patch }} V \cdot D=\frac{N_{\text {patch }}}{2} \sum_{\mu=z, t}\left[U_{\mu}(x) \delta_{x, y-\mu}-U_{\mu}^{\dagger}(x-\mu) \delta_{x, y+\mu}\right]
$$

where $V=(1, \vec{v})$. The NLO term can be written as

$$
M_{\mathrm{NLO}}=\frac{N_{\mathrm{patch}}}{2 \mu}\left(D_{x}^{2}+D_{y}^{2}+i \sigma_{z}\left[D_{x}, D_{y}\right]\right)
$$

where $\sigma_{z}$ is the usual Pauli matrix. 


\section{Discussion}

The LO operator that is used to generate the configurations has been tested for reversibility and linearity of $\Delta H$ with the microcanonical step size squared. Reweighting in the NLO term has also been incorporated and measurements are under way. The HDET code has been adapted from the MILC code [9]. One remaining issue in the implementation of HDET on the lattice is the inclusion of the Debye screening mass term which should be included in order to match to the full theory [10]. Since this contribution is not gauge invariant, it is not clear how this term can be included in the generation of dynamical lattices and more work must be carried out in this area.

In summary, we have given a brief overview of the QCD phase diagram and highlighted the necessity of finding an alternative approach to QCD at mid to high density on the lattice. We have reviewed the principle features of high density effective theory and presented our strategy for implementing this theory on the lattice. The aim of this work is to look for evidence of the densitydriven phase transition. It is not clear at this stage how low it is possible to take the chemical potential before the breakdown of the expansion in the effective theory, however, we are guided by the fact that provided the residual momentum $l$ which is $O\left(\Lambda_{\mathrm{QCD}}\right)$ is much smaller than the chemical potential, ie. $l / \mu<<1$, the expansion should be valid.

\section{Acknowledgments}

I am very grateful to Zoltan Fodor, who proposed this project, Deog Ki Hong, Craig McNeile, Kalman Szabo and Fermin Viniegra for many useful discussions.

\section{References}

[1] Y. Aoki et al, The order of the quantum chromodynamics transition predicted by the standard model of particle physics, Nature 443 (2006) 675 [hep-lat/0611014].

[2] Y. Aoki et al, The QCD transition temperature: Results with physical masses in the continuum limit, Phys. Lett. B 643 (2006) 46 [hep-lat/0609068].

[3] Z. Fodor and S. Katz, Critical point of QCD at finite T and mu, lattice results for physical quark masses, JHEP 0404 (2004) 050 [hep-lat/0402006].

[4] Z. Fodor et al, The Density of states method at non-zero chemical potential, JHEP 0703 (2007) 121 [hep-lat/0701022].

[5] Mark G. Alford et al, Color-flavor locking and chiral symmetry breaking in high density QCD, Nucl. Phys. B 537 (1999) 443 [hep-lat/9804403].

[6] Deog Ki Hong, An effective field theory of QCD at high density, Phys. Lett. B 473 (2000) 118 [hep-ph/9812510].

[7] Deog Ki Hong and Stephen D. H. Hsu, Positivity of high density effective theory, Phys. Rev. D 66 (2002) 071501 [hep-ph/0202236].

[8] Z. Fodor and S. D. Katz, Lattice determination of the critical point of QCD at finite T and mu, JHEP 03 (2002) 014 [hep-lat/0106002].

[9] MILC Collaboration, public lattice gauge theory code, see http://physics.indiana.edu/sg/milc.html.

[10] Deog Ki Hong, Color superconductivity and high density effective theory, Prog. Theor. Phys. Suppl. 153 (2004) 241 [hep-ph/0401057]. 